\section{AB0501 1 THREE CASES OF VERTEBRAL ARTERITIS IDENTIFIED ON FDG-PET IN PATIENTS WITH SUSPECTED GCA AT UNIVERSITY COLLEGE LONDON HOSPITAL (UCLH)}

D. Ludwig ${ }^{1}$, M. Naja ${ }^{1}$, S. Voo ${ }^{1}$, V. Morris ${ }^{1} .{ }^{1}$ University College London Hospital, London, United Kingdom

Background: Giant cell arteritis (GCA) may affect both cranial and extra-cranial vessels; where the latter occurs, it can be termed large-vessel GCA (LV-GCA). Large vessel involvement is common: histological evidence has been seen in $80 \%$ of autopsies of patients with known GCA, and imaging studies suggest large vessel involvement in over $80 \%{ }^{1}$. LV-GCA is important to diagnose due to the risks of vascular complications such as occlusion and ischaemic stroke. The clinical diagnosis can be challenging, and the American College of Rheumatology (ACR) GCA classification criteria often underperform in cases of LV-GCA ${ }^{1}$. F-fluorodeoxyglucose positron emission tomography (FDG-PET) has been found to be useful in the detection of extra-cranial involvement to support the diagnosis of LV-GCA. ${ }^{2}$ Objectives: To appreciate the variability in presentation of cases of LV-GCA, and to further characterise a subgroup of patients with vertebral arteritis.

To explore the use of FDG-PET imaging in GCA patients in addition to or in place of traditional diagnostic tools (temporal artery ultrasound / biopsy).

Methods: Through evaluation of the new GCA fast-track pathway implemented at $\mathrm{UCLH}$, a subgroup of patients diagnosed with vertebral arteritis was identified. The history and presentation of these patients were analysed.

Results: Three patients were diagnosed with vertebral arteritis. All three were male, Caucasian and aged over 70. All were investigated for GCA due to a history of severe headache (frontal in one, occipital in one, bi-temporal in one) with associated red flag symptoms. Two had a history of jaw claudication and visual disturbances (unilateral visual loss in one, transient diplopia in the other). Both of these patients had positive temporal artery biopsies. The third patient had no ischaemic symptoms but a strong history of prominent polymyalgic features and a positive temporal artery ultrasound. Inflammatory markers were raised in two, and normal in one, of the patients. Only one had systemic symptoms (weight loss). All three proceeded to FDG-PET scans which showed vertebral arteritis and were commenced on immunosuppressive treatment. Conclusion: The cases discussed illustrate the heterogeneity of the presentation of LV-GCA, and the diagnostic challenge this poses. FDG-PET imaging is useful in confirming extra-cranial involvement and therefore guiding treatment. References:

[1] Large-vessel giant cell arteritis: diagnosis, monitoring and management. Matthew J Koster, Eric L Matteson, Kenneth J Warrington. 2018, Rheumatology, Vol. 57, pp. 32-42.

[2] EULAR recommendations for the use of imaging in large vessel vasculitis in clinical practice. Dejaco C, Ramiro S, Duftner C, et al. 2018, Annals of the Rheumatic Diseases, Vol. 77, pp. 636-643.

Disclosure of Interests: : None declared

DOI: 10.1136/annrheumdis-2020-eular.410

\section{AB0502 \\ EVALUATION OF A NOVEL FAST TRACK PATHWAY FOR GIANT CELL ARTERITIS USING PET IN ADDITION TO TAUS ANDTAB FOR EARLY DIAGNOSIS}

D. Ludwig ${ }^{1}$, A. Shashidhara ${ }^{1}$, C. Stavrou ${ }^{1}$, S. Voo ${ }^{1}$, R. Reshat ${ }^{1}$, V. Morris ${ }^{1}$.

${ }^{1}$ University College London Hospital, London, United Kingdom

Background: : Giant Cell Arteritis (GCA) is a common primary systemic vasculitis (1). Its predilection for the temporal artery can result in permanent visual loss if left untreated. Over $25 \%$ of patients have involvement of large vessels, such as the aorta, resulting in an increased risk of aneurysm formation and dissection. (3) Diagnosis of GCA is largely clinical and temporal artery biopsy (TAB) has long been the gold standard for diagnosis. In recent years temporal artery ultrasound (TAUS) has emerged as an effective, non-invasive tool to aid diagnosis. Positron-emission tomography (PET) can also be utilised to detect the presence of large vessel involvement but is currently not used in many centres for diagnostic purposes and requires further standardisation and validation (2). The challenge arises from these investigations losing sensitivity in the days following steroid treatment, meaning that rapid access is key to confirm diagnosis.

Objectives: To evaluate the impact of the introduction of a fast track pathway (FTP) on prompt diagnostics and treatment.

To improve our understanding of the use of PET at diagnosis and compare this to the gold standard TAB, and to TAUS, in our institution.

Methods: Cohort 1: 32 patients, all presenting before FTP implementation, identified from outpatient clinics and referrals to the Rheumatology team. Time taken from steroid initiation to TAUS/TAB was extracted from clinical records. Outcomes for this group included number of years on steroid (steroid burden).

Cohort 2: 21 patients all referred after implementation of a new GCA FTP. This group contains all patients referred as query GCA, not just those with positive diagnoses. Time from steroid initiation to TAUS/TAB was recorded. The FTP included the addition of PET imaging within 72 hours.
Results: Cohort 1: 20 (63\%) patients had TAB and $3(9 \%)$ had TAUS. The avergige time from starting steroid to investigation was 5.2 days and 2 days respectively. The average steroid burden in patients with no confirmatory test was 11 years. If patients had just a single diagnostic test this value dropped to 3 years. Cohort 2: 11 (52\%) had TAB, 10 (48\%) had TAUS and 11 (52\%) had PET. In postive GCA diagnoses, time from steroid start to investigations was 7.2 days, 1 day and 3.2 days respectively. In patients with a negative diagnosis the time frames were 13 days, 1 day and 1.7 days respectively. Sensitivity for TAB was $45.5 \%$ and TAUS $40 \%$. Specificity for TAB and TAUS was $100 \%$. These results are comparable to similar studies (4). PET sensitivity was $63.6 \%$ and specificity $100 \%$.

Table 1. Sensitivity, specificity, predictive values

\begin{tabular}{llll}
\hline & TAB & TAUS & PET CT \\
\hline Sensitivity (\%) & 45.5 & 40.0 & 63.6 \\
Specificity (\%) & 100 & 100 & 100 \\
PPV (\%) & 100 & 100 & 100 \\
NPV (\%) & 33.3 & 57.1 & 55.6 \\
\hline
\end{tabular}

Conclusion: Prompt diagnostics, best facilitated through a FTP, can reduce steroid burden in GCA even if only one confirmatory test is available. Patients with a low clinical suspicion of GCA and negative TAUS or a high clinical suspicion and positive TAUS stand little to gain from TAB. These findings summarise the first 6 months of our GCA FTP. Continued evaluation of PET in our FTP is needed to understand its role in diagnosis, particularly in patients at risk of vascular complications. References:

[1]Crowson CS, Matteson EL. Contemporary prevalence estimates for giant cell arteritis and polymyalgia rheumatica 2015. Semin Arthritis Rheum. 2017; 47 (2) 253-6

[2] Camellino D, Dejaco C. Should 18 F FDG PET imaging be used in the diagnosis of GCA? Nature Reviews Rheumatology 2019; 15 (7) 388-90

[3]Nuenninghoff DM et al. Incidence and predictors of large-artery complication (aortic aneurysm, aortic dissection, and/or large artery stenosis) in patients with giant cell arteritis: a population-based study over 50 years. Arthritis Rheum 2003; 48:3522-3531

[4] Luqmani, R. et al. The Role of Ultrasound Compared to Biopsy of Temporal Arteries in the Diagnosis and Treatment of Giant Cell Arteritis (TABUL): A Diagnostic Accuracy and Cost-Effectiveness Study. Health Technol Assess 2016; 20 (90) 1-238

Disclosure of Interests: None declared

DOI: 10.1136/annrheumdis-2020-eular.3957

\section{AB0503 USE OF BIOLOGICAL THERAPY IN A COHORT OF PATIENTS WITH TAKAYASU ARTERITIS IN A THIRD LEVEL HOSPITAL.}

R. J. Gil Velez ${ }^{1}$, E. Rubio Romero ${ }^{1}$, I. Madroñal García ${ }^{1} .{ }^{1}$ Hospital Universitario Virgen del Rocío, Reumatologia, Sevilla, Spain

Background: Takayasu arteritis is a granulomatous vasculitis that affects large vassels, specially aorta and its main ramifications. It is a rare disease with an incidence between 0.3 and 1.2 per million inhabitants in Europe. It is more common in women (80\%) and it usually occurs before age 40

Objectives: Describe the characteristics and the use of biological therapy in a cohort of patients with Takayasu arteritis in our Hospital.

Methods: Retrospective descriptive study of patients diagnosed with Takayasu arteritis treated in the Rheumatology and Internal Medicine service in our hospital during 2019. Data have been obtained by reviewing medical records.

Results: 39 patients with takayasu arteritis were included, 33 women (84.4\%) and 6 men, withan average age of $48 \pm 12$ years. About treatment, 35 patients received glucocorticoid treatment, of these, 23 patients $(65.7 \%)$ received treatment with DMARDs. Methotrexato (47.8\%) and Ciclophosphamide (43.5\%) are the most used. $12.8 \%(5)$ of patients received treatment with biological therapy 2 patients received treatment with Rituximab (already retired) and 3 patients maintained the treatment with Tocilizumab. (The characteristics of the patients are in table 1). About the course of disease, $47.8 \%$ of patients have achieved clinical remission, $43.6 \%$ have a chronic course, and only 3 patients have presented relapse of disease.

Conclusion: The results of our study are consistent with what is published in the lit-

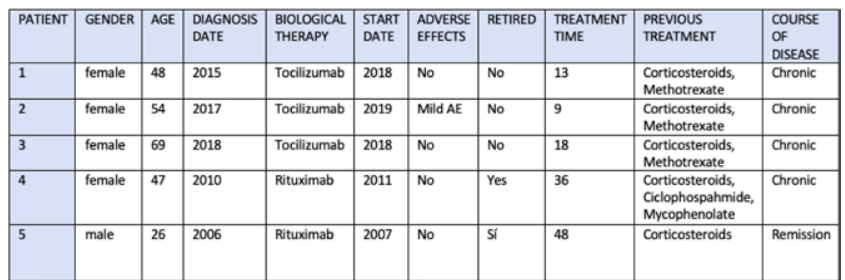

erature. The average age of presentation is higher than in other series ( $48 \pm 12$ years), this is because all patients are European and Caucasian. There is still not much experience on biological treatment in patients diagnosed with sarcoidosis, however the 
use of Rituximab, AntiTNFs and Tocilizumab has been described, presenting a good clinical response and with few adverse effects, as has been observed in our patients. Disclosure of Interests: None declared

DOI: 10.1136/annrheumdis-2020-eular.6074

\section{AB0504 THE DIFFERENCES OF IMMUNE CHARACTERISTICS AND ITS RELATIONSHIP WITH DISEASE ACTIVITY IN LARGE VESSEL VASCULITIS PATIENTS}

A. Maimaitiyakefu ${ }^{1}$, L. Wu ${ }^{1} .{ }^{1}$ Department of Rheumatology and Immunology in People's Hospital of Xinjiang Uygur Autonomous Region, China, Urumqi, Xinjiang, China

Background: Large vessel vasculitis(LVV) is a rare refractory disease, giant cell arteritis (GCA) and Takayasu arteritis (TAK) constitute different types of primary LVV. Despite considerable discussion on the potential differences between GCA and TAK, the immunological difference and its relationship to disease activity remains unclear. Objectives: To investigate the differences of peripheral blood immuno-phenotype between TAK and GCA, and to analyze the correlation between immune metabolism level and disease activity in TAK.

Methods: All patients with TAK and GCA who come to our department from January 2010 to January 2020 were included, all of them met the classification criteria of American college of rheumatology.General information, clinical manifestations, laboratory index, artery involvement and therapeutic schedule were collected.According to the National Institutes of Health criteria, there were 29 active and 23 inactive TAK patients. Results:

[1] Baseline characteristics, treatment,involvement artery in LVV patients are summarized in Table 1.

Table 1. Clinical characteristics of patients with LVV

\begin{tabular}{lcc}
\hline Variable & TAK,n=52 & GCA,n=4 \\
\hline Age, $\mathrm{X} \pm \mathrm{S}$ & $29 \pm 13$ & $64 \pm 7$ \\
Female,n(\%) & $45(86.6)$ & $1(25)$ \\
Constitutional symptoms,n(\%) & $23(43.4)$ & $3(75)$ \\
Cephalic symptoms,n(\%) & $33(62.3)$ & $2(50)$ \\
Peripheral limb ischemia,n(\%) & $22(41.5)$ & - \\
Ocular signs,n(\%) & $3(5.7)$ & $2(50)$ \\
Stroke,n(\%) & $2(3.8)$ & - \\
GC,n(\%) & $40(76.9)$ & $3(75)$ \\
GC dose $>7.5 m g / d a y, n(\%)$ & $36(90)$ & $2(66.7)$ \\
Surgical intervention,n(\%) & $7(13.2)$ & - \\
Coronary artery,n(\%) & $3(5.7)$ & - \\
Common carotid artery,n(\%) & $31(58.5)$ & - \\
Subclavian artery,n(\%) & $23(43.4)$ & - \\
thoracic aorta,n(\%) & $6(11.3)$ & - \\
abdominal aorta,n(\%) & $13(24.5)$ & - \\
\hline
\end{tabular}

GC Glucocorticoids,bDMARDS biologic DMARDs

(2) Compared with GCA patients, TAK patients were younger $(29 \pm 13 v s 64 \pm 7, \mathrm{P}=0.015)$, with higher peripheral blood lymphocyte count of CD8+T cells and lower CD4/CD8(Table 2).

Table 2. Comparison of peripheral immune cells, humoral immunity among TAK and GCA, active and inactive TAK

\begin{tabular}{|c|c|c|c|c|c|c|}
\hline Variable & $\mathrm{TAK}, \mathrm{n}=52$ & GCA, $n=4$ & $\begin{array}{c}p \\
\text { value }\end{array}$ & $\begin{array}{c}\text { inactive } \\
\text { TAK, } n=23\end{array}$ & $\begin{array}{c}\text { active } \\
\text { TAK,n=29 }\end{array}$ & $\begin{array}{c}\mathbf{p} \\
\text { value }\end{array}$ \\
\hline Neutrophils & $5.5 \pm 2.3$ & $5.8 \pm 1.1$ & 0.827 & $4.4 \pm 1.6$ & $6.3 \pm 2.5$ & $0.009^{*}$ \\
\hline Lymphocytes & $2.4 \pm 0.8$ & $1.0 \pm 0.6$ & $0.010^{*}$ & $2.3 \pm 0.7$ & $2.4 \pm 0.9$ & 0.901 \\
\hline Monocytes & $0.5 \pm 0.2$ & $0.7 \pm 0.1$ & 0.218 & $0.5 \pm 0.2$ & $0.6 \pm 0.2$ & 0.431 \\
\hline $\mathrm{CD}^{+} \mathrm{T}^{-}$ & $1959 \pm 490$ & $1154 \pm 541$ & 0.189 & $2195 \pm 92$ & $1842 \pm 585$ & 0.467 \\
\hline $\mathrm{CD} 4^{+} \mathrm{T}$ & $1074 \pm 305$ & $758 \pm 303$ & 0.381 & $1250 \pm 198$ & $987 \pm 333$ & 0.376 \\
\hline $\mathrm{CD}^{+} \mathrm{T}$ & $859 \pm 212$ & $297 \pm 87$ & $0.048^{*}$ & $945 \pm 41$ & $815 \pm 258$ & 0.542 \\
\hline $\mathrm{CD}^{+} \mathrm{CD}^{+}$ & $42 \pm 27$ & $12 \pm 7$ & 0.344 & $63 \pm 2$ & $32 \pm 28$ & 0.229 \\
\hline CD4-CD8- & $90 \pm 75$ & $91 \pm 63$ & 0.999 & $97 \pm 74$ & $87 \pm 68$ & 0.914 \\
\hline $\mathrm{CD} 19^{+} \mathrm{B}$ & $273 \pm 97$ & $191 \pm 94$ & 0.470 & $202 \pm 106$ & $309 \pm 84$ & 0.242 \\
\hline $\mathrm{CD}_{16}{ }^{+} \mathrm{CD} 56^{+}$ & $135 \pm 72$ & $104 \pm 67$ & 0.709 & $112 \pm 63$ & $146 \pm 83$ & 0.639 \\
\hline CD4/CD8 & $1.25 \pm 0.15$ & $2.55 \pm 0.51$ & $0.001^{*}$ & $1.33 \pm 0.26$ & $1.21 \pm 0.10$ & 0.440 \\
\hline $\lg G$ & $11.7 \pm 3.7$ & $8.3 \pm 0.5$ & 0.124 & $10.3 \pm 3.4$ & $12.9 \pm 3.6$ & $0.025^{*}$ \\
\hline $\lg A$ & $2.6 \pm 1.3$ & $2.3 \pm 0.8$ & 0.724 & $2.1 \pm 1.3$ & $3.0 \pm 1.1$ & $0.021 *$ \\
\hline $\operatorname{lgM}$ & $1.7 \pm 0.9$ & $0.8 \pm 0.2$ & 0.118 & $1.5 \pm 0.8$ & $1.8 \pm 0.9$ & 0.202 \\
\hline $\mathrm{K}$ light chain & $9.5 \pm 3.9$ & $6.6 \pm 0.3$ & 0.222 & $8.1 \pm 3.3$ & $10.8 \pm 4.0$ & $0.033^{*}$ \\
\hline$\lambda$ light chain & $5.2 \pm 1.9$ & $3.9 \pm 0.3$ & 0.260 & $4.7 \pm 1.9$ & $5.8 \pm 1.9$ & 0.086 \\
\hline
\end{tabular}

"P $<0.05$

(3)The levels of neutrophils, monocytes, IgG, IgA and $K$ light chain active TAK were higer than inactive TAK, with lower lymphocyte level (Table 2). Further correlation analysis showed that the levels of neutrophils, IgG, IgA and $K$ light chain were positively correlated with disease activity $(r=0.434, P=0.005 ; r=0.380, P=0.019 ; r=0.452, P=0.004 ; r=0.380, P=0.022)$.
Conclusion: Compared with GCA patients, TAK patients had higer level of CD8 ${ }^{+} \mathrm{T}$ lymphocytes, consistenting with previous studies.In addition, TAK patients had persistent immune cell involvement, while humoral immunity is related to disease activity.Deeper studies may be required about the role of immune profile in in large vessel vasculitis.

References:

[1] Miyabe $\mathrm{C}$, Miyabe $\mathrm{Y}$, et al. An expanded population of pathogenic regulatory T-cells in giant cell arteritis is abrogated by IL-6 blockade therapy. Ann Rheum Dis. 2017;76:898-905.

[2] Kermani TA, Crowson CS, Muratore F, Schmidt J, Matteson EL, Warrington KJ. Extra-cranial giant cell arteritis and Takayasu arteritis: how similar are they? Semin Arthritis Rheum. 2015;44:724-8.

Disclosure of Interests: None declared

DOI: 10.1136/annrheumdis-2020-eular.4126

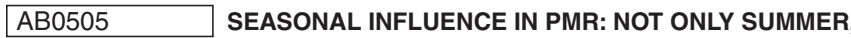 BUT WINTER IS COMING TOO}

D. Marsman ${ }^{1}$, N. Den Broeder ${ }^{2}$, F. Van den Hoogen ${ }^{2}$, A. Den Broeder ${ }^{2}$, A. Van der Maas ${ }^{3} .{ }^{1}$ Sint Maartenskliniek, Rheumatology, Ubbergen, Netherlands; ${ }^{1}$ Sint Maartenskliniek, Rheumatology, Ubbergen, Netherlands; ${ }^{3}$ Sint Maartenskliniek, Ubbergen, Netherlands

Background: The cause for polymyalgia rheumatica (PMR) is currently unknown. Disease onset may be triggered by a combination of genetic predisposition and environmental factors such as infection. ${ }^{1}$ In different regions of Denmark a simultaneous peak incidence of giant cell arthritis and PMR occurred together with epidemics of Mycoplasma pneumoniae, Chlamydophila pneumoniae and Parvovirus B19. ${ }^{1}$ A seasonal epidemics pattern for PMR would be supporting evidence for an infectious cause. ${ }^{1}$ However, the current evidence of seasonal effect on the occurrence and disease severity of PMR is limited and show conflicting results. ${ }^{2,3}$

Objectives: To evaluate whether there is a seasonal effect on the risk of developing PMR in the Netherlands

Methods: We retrospectively collected data on patient-, disease,- and treatment characteristics from newly diagnosed PMR patients (clinical diagnosis) who visited our outpatient clinic during April 2012 and September 2017. Exclusion criteria was other concomitant inflammatory rheumatic disease. Based on the onset of PMR (start symptoms, not time of diagnosis) patients were grouped per month. Descriptive statistics were used [mean (SD), median (p25-p75) or $n$ (\%) as appropriate]. The Chi square goodness of fit test was used to determine whether the incidence of onset of symptoms was different between months of the year.

Results: In total 448 patients were included and $55 \%$ were female and mean age was 66 years. Other baseline characteristics are described in table 1. The chi-square goodness of fit test to determine whether there was a peak incidence in months was $p=0.06$. As shown in figure 1 the incidence of onset PMR symptoms is higher in December-January, April through June with a peak in August. The April-June peaks coincides with incidence peaks of Mycoplasma pneumoniae infections and possibly Parvovirus B19 in spring and summer, the December-January peak coincides with Parvovirus B19 infections. ${ }^{4,5}$

Table 1. Baseline characteristics $(n=448)$

Characteristic

Female (\%)

Age, years (SD)

PMR symptoms before diagnosis, weeks (IQR)

Neck pain (\%)

Bilateral shoulder pain

/stiffness (\%)

Bilateral hip pain/stiffness (\%)

Morning stiffness $>45 \mathrm{~min}(\%)$

Peripheral arthritis (\%)

Systemic symptoms* $(\%)$

Elevated ESR mm/hour and / or CRP mg/l

ESR mm/hour(IQR)

CRP mg/l (IQR)

$247(55)$

$66(8.6)$

$10(6-16)$

205 (46)

$412(91)$

$380(85)$

$233(52)$

$35(8)$

199 (44)

$37(26-51)$

${ }^{*}$ Fever, night sweats, weight loss, anorexia

** ESR $n=428 ;$ CRP $n=396$

Conclusion: No definitive seasonal effect was found on risk of developing PMR although a bimodal seasonal pattern compatible with the proposed respiratory infections is suggested.

References:

[1] González-Gay MA et al.Polymyalgia rheumatica. Lancet. 2017 Oct Oct 7. 